\title{
A Novel Vibration Control System Applying Annularly Arranged Thrusters for Multiple Launch Rocket System in Launching Process
}

\author{
Lilin Gu, Xiaoting Rui ${ }^{D}$, Guoping Wang, Fufeng Yang, and Min Wei \\ Institute of Launch Dynamics, Nanjing University of Science and Technology, Nanjing, Jiangsu 210094, China \\ Correspondence should be addressed to Xiaoting Rui; ruixt@163.net
}

Received 21 February 2020; Revised 1 May 2020; Accepted 22 May 2020; Published 11 June 2020

Academic Editor: Shuaishuai Sun

Copyright ( $\odot 2020$ Lilin Gu et al. This is an open access article distributed under the Creative Commons Attribution License, which permits unrestricted use, distribution, and reproduction in any medium, provided the original work is properly cited.

\begin{abstract}
Multiple Launch Rocket System (MLRS) has been widely used in recent years; vibration control in launching process is an effective way to improve its dispersion characteristics. In this paper, a novel vibration control system applying Annularly Arranged Thrusters (AAT) for MLRS in launching process is introduced and the prototype of the proposed system is built. The dynamic model of the MLRS with the AAT is established based on the Transfer Matrix Method for Multibody Systems (MSTMM). The LQR-PID control law and the management for the AAT are presented. The simulation and experiment of the proposed system are carried out and analyzed. The results show that the vibration of MLRS is effectively attenuated by the proposed control system. The study in this paper provides a new idea to improve the dispersion characteristic by reducing the vibration of MLRS in launching process.
\end{abstract}

\section{Introduction}

Multiple Launch Rocket System (MLRS) has been widely used all around the world due to the advantages such as high firing speed, large firing area, long firing range, good mobility, and heavy firepower density weaved in a short period of time. Dispersion characteristic is one of the important factors affecting its development [1]. The MLRS produces vibration in launching process (starts when a rocket moves and finishes when its rear band leaves the launch tube) because of the jet force of the last rocket and the contact force between the rocket pilot pin and the launch tube helical groove. This vibration will change the attitude of MLRS, while the rockets are moving in the launch tubes, then affecting the initial disturbance of the rockets, and thus influencing the dispersion characteristics [2].

The earliest research about the vibration control for the MLRS was started with "passive control," which was presented by Cochran et al. [3-5]. They attempted to counteract the ballistic deflection due to rocket imperfections with the ballistic deflection due to the initial disturbance by optimizing system parameters. Chen and Zhao [6] discussed the physics model of the rocket passive controller and simulated results showed that the passive controller was not affected by the randomness of the initial direction of the rocket's errors. Zhao and Chen [7] used elastic sabots as a passive controller and the dispersion due to thrust misalignment and dynamic unbalance was reduced. Zhang et al. [8] treated the passive control problem as the trajectory planning problem of a given part which can be solved by changing parameters. The control equations were established by Driver Constraint Method. However, the passive control method is only applicable to the vibration caused by the rocket's imperfections, not to the vibration caused by the jet force. Moreover, the vibration response can only be controlled up to a certain limit due to the lack of energy.

With the development of equipment and the advance in technology, continuous researches have been focused on the active or semiactive control of MLRS to improve the dispersion characteristics. Xu et al. [9] studied the azimuth part and the elevation part which were, respectively, driven by the hydraulic motor and the hydraulic cylinder. They provided a 
variable rigidity and damp dynamic response control, which changes the vibration characteristic of launcher system by on-off strategy. Inspired by the nonlinear computed torque approach which is widely used in robotic manipulators, Dokumaci et al. [10] designed a PID feedback control for the servo system and applied it to the launcher system. Based on the work of Dokumaci, Li and Rui [11] considered the coupling model of the launcher system and the servo motor and improved the computed torque approach by radial basis function neural network algorithm, which effectively controlled the vibration of MLRS with uncertainty. However, there are two common shortcomings in these researches. Firstly, the launching process of rockets is $120 \mathrm{~ms}$ in actual applications when the stochastic input delay for the hydraulic cylinder and servo system will reach hundreds of milliseconds. The control commends cannot be reacted timely in these control systems, resulting in the fact that the vibration of MLRS in launching process cannot be reduced in these systems. Only the attitude of MLRS when the next rocket begins to launch can be controlled by avoiding the jet force's influence. Secondly, MLRS is a complex multirigidflexible system in reality, while the dynamic models of MLRS used in the above researches are very simple, only considering the azimuth part and the elevation part as rigid bodies. Because the simple model is far different from reality, neither is the motion of MLRS described exactly, nor the design of the controller is convinced. Hence, the fast-reacted actuator and the accurate model are important issues to be considered in the vibration control of MLRS in launching process.

The thruster is a device that converts the gas generated by the combusting charge to the output force through the nozzle. It has advantages such as fast response speed, large output force, and easy to control and has been widely used in missile guidance, spacecraft attitude control, satellite vibration control, and projectile attitude correction. Li and Qi [12] proposed a logic-based guidance strategy to improve the homing performance of a low-altitude endoatmospheric interceptor. Yang and Gao [13] investigated the problem of robust reliable control for the spacecraft rendezvous with limited thrust. Lim [14] designed the phase-plant controller for the thruster attitude control system of the Tactical Satellite 4 to abate the undesirable effects by employing hysteresis in the switching lines. Zhan et al. [15] took a brief attempt to apply the thrusters to control the vibration of MLRS caused by the jet force, only considering the single shot condition. Gu et al. [16] discussed the vibration control of MLRS with thrusters in launching process under simple simulated conditions.

The Transfer Matrix Method for Multibody Systems (MSTMM) [17-20] is a new method for multibody system dynamics in recent 20 years, which studies multibody system dynamics through the transfer relationship between the state vectors in multibody system. It has advantages such as no need for global dynamic equations of the system, the lower order of system matrix, high programming, high precision, and suitable for modeling and controller designing for complex multibody systems. Wang et al. [21] presented a robust controller using the independent modal space control method for active vibration control of general linear multibody. Chen et al. [22] studied the model of the Stewart parallel mechanism and employed LQR control. Rong et al. [23] researched the dynamics modeling and analysis of the ship's seaborne supply system and designed a hybrid control system composed of three PD controllers and a fuzzy controller to compensate the ship's relative motion caused by sea waves.

In summary, applying the thrusters as actuators and modeling through the MSTMM are meaningful to the vibration control of MLRS in the short launching process. For the above purpose, a novel vibration control system with the Annularly Arranged Thrusters (AAT) is designed in this paper. The rest of this paper is organized as follows. The AAT is designed in Section 2. The dynamic model of the MLRS with AAT is established in Section 3. In Section 4, the vibration control system in launching process for the MLRS with the AAT is proposed. The simulation system is established and results are analyzed in Section 5. The experiment and analysis are presented in Section 6. Finally, the conclusion is included in Section 7.

\section{The Model and Design of the AAT}

2.1. The Mathematical Model of the Thruster. Thrusters are only capable of providing approximately fixed-magnitude $F_{P}$ impulse forces during a short-duration $\Delta t_{P}$. However, a thruster force cannot reach its full value discontinuously in practice. Based on the experimental result, a continuous smooth mathematical model of the thruster is derived by fitting the sinusoidal curve in Figure 1. $\Delta t_{r}$ and $\Delta t_{d}$ are assumed to be the rise time from the start time to reach its full value and decay time to reach its end time, respectively. And the duration $\Delta t_{P}=\Delta t_{r}+\Delta t_{f}+\Delta t_{d}$.

$$
f_{P}(t)= \begin{cases}\frac{1}{2} F_{P}\left[1-\cos \frac{\pi\left(t-t_{s}\right)}{\Delta t_{r}}\right], & t_{s} \leq t \leq t_{s}+\Delta t_{r}, \\ F_{p}, & t_{s}+\Delta t_{r} \leq t \leq t_{s}+\Delta t_{p}-\Delta t_{d}, \\ \frac{1}{2} F_{P}\left[1-\cos \frac{\pi\left(t-t_{s}-\Delta t_{p}\right)}{\Delta t_{d}}\right], & t_{s}+\Delta t_{p}-\Delta t_{d} \leq t \leq t_{s}+\Delta t_{p} .\end{cases}
$$




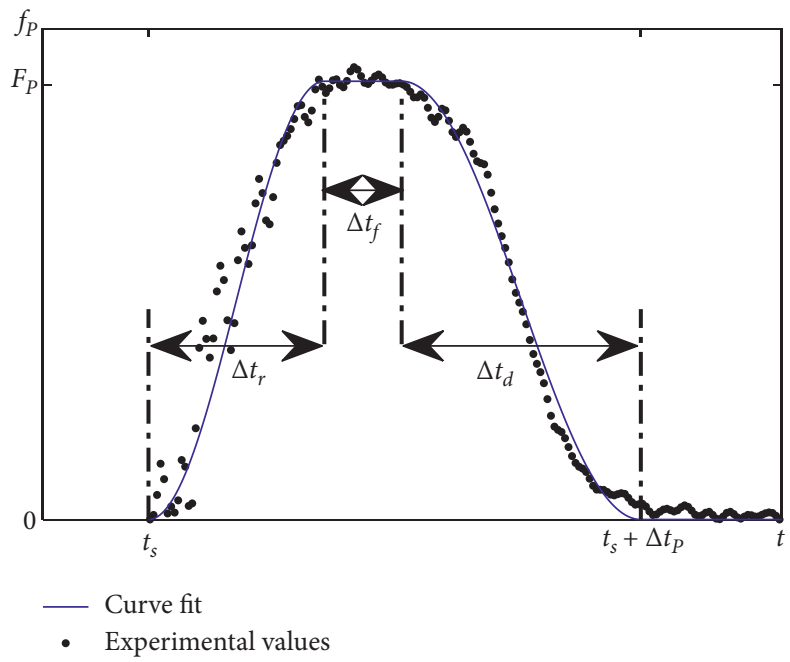

FIGURE 1: The mathematical model of the thruster.

2.2. The Design of the AAT. The AAT is designed as shown in Figure 2. An annular base is mounted on the tip of the elevation part and thrusters are arranged in a uniform sequence on the base.

As shown in Figure 2(b), the annular base's center is denoted as $O_{P}\left(l_{P x}, l_{P y}, 0\right)$ in the body-fixed frame of the elevation part $O_{23, I} x_{23, I} y_{23, I} z_{23, I}$. The body-fixed frame of the annular base is denoted as $O_{P} x_{P} y_{P} z_{P}$ and parallel to $\mathrm{O}_{23, I} x_{23, I} y_{23, I} z_{23, I}$. The radius of the annular base is $r_{p}$ and the thrusters on the base are numbered counterclockwise from 1 to $N$ (rear view) in Figure 3. The application point and the associated components of the $n^{\text {th }}$ thruster in the along the body-fixed frame $O_{23, I} x_{23, I} y_{23, I} z_{23, I}$ come as

$$
\begin{aligned}
l_{O_{23, I} O_{P_{n}}} & =\left(l_{P x}, l_{P y}+r_{P} \sin \left(\frac{n-1}{N} \cdot 2 \pi\right), r_{P} \cos \left(\frac{n-1}{N} \cdot 2 \pi\right)\right)^{\mathrm{T}}, \\
F_{P_{n}} & =\left(F_{x_{n}}, F_{y_{n}}, F_{z_{n}}\right)=\left(0,-f_{P} \sin \left(\frac{n-1}{N} \cdot 2 \pi\right),-f_{P} \cos \left(\frac{n-1}{N} \cdot 2 \pi\right)\right)^{\mathrm{T}} .
\end{aligned}
$$

Then, the force and its moment according to the thruster force in $y$ and $z$ direction in $O_{23, I} x_{23, I} y_{23, I} z_{23, I}$, which are denoted as $F_{y_{n}}$ and $F_{z_{n}}$, are deduced as

$$
f_{P_{n}}=\left[\begin{array}{c}
F_{P_{n}} \\
l_{O_{23, I} O_{P_{n}}} \times F_{P_{n}}
\end{array}\right]=\left[\begin{array}{cccccc}
0 & 1 & 0 & 0 & 0 & l_{p x} \\
0 & 0 & 1 & l_{p y} & -l_{p x} & 0
\end{array}\right]^{\mathrm{T}}\left[\begin{array}{c}
F_{y n} \\
F_{z n}
\end{array}\right] \text {. }
$$

In such form, the action of $n^{\text {th }}$ thruster is associated only with the thruster force, while the position of annular base is fixed in engineering applications.

\section{Dynamic Modeling of MLRS with the AAT}

3.1. The Dynamic Model of the MLRS. The MLRS is mainly composed of the rear mechanical jacks and the wheels, the vehicle chassis, the azimuth part, the elevation part, launch tubes, and the AAT. Thrusters act only on the elevation part. The mass of the AAT is incorporated into the elevation part and the mass properties variations of the elevation part due to the action of thrusters are ignored.

According to MSTMM [18], the body elements and hinge elements are numbered uniformly shown in
Figure 4(a). The rear mechanical jacks and the wheels, which are both considered as rigid bodies, are numbered as 2, 5, 8, 11,14 , and 17. The vehicle chassis, the azimuth, and elevation part including the thrusters are regarded as spatial rigid body elements 19,21 , and 23, respectively. Launch tubes are divided into three parts and numbered as $26+5 l, 27+5 l$, and $28+5 l$, respectively, where $l(l=1,2, \ldots, 18)$ denotes the number of the launch tubes. Body elements $26+5 l$ are considered as spatial rigid bodies, while body elements $27+5 l$ and $28+5 l$ are considered as elastic beams transversely vibrating in space. The actions between body elements mentioned above and their elastic and damping effect are modeled as parallel rotary springs, linear springs, and dampers in 3 directions. The hinges are numbered as $1,3,4$, $6,7,9,10,12,13,15,16,18,20,22,24+5 l$, and $25+5 l$. There are 8 boundary ends numbered as 0 . In summary, the MLRS is a multirigid-flexible system, composed of 27 rigid bodies and 36 flexible bodies connected with various linear springs, rotary springs, and dampers, respectively.

3.2. The Overall Transfer Matrix and the Characteristic Equation. The topology describes the relationship among the state vectors of elements and the transfer directions, 


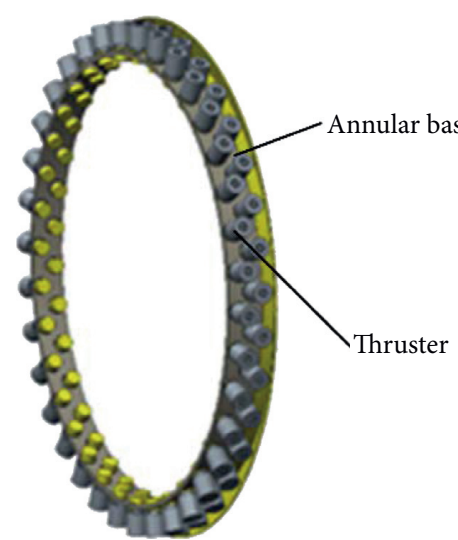

(a)

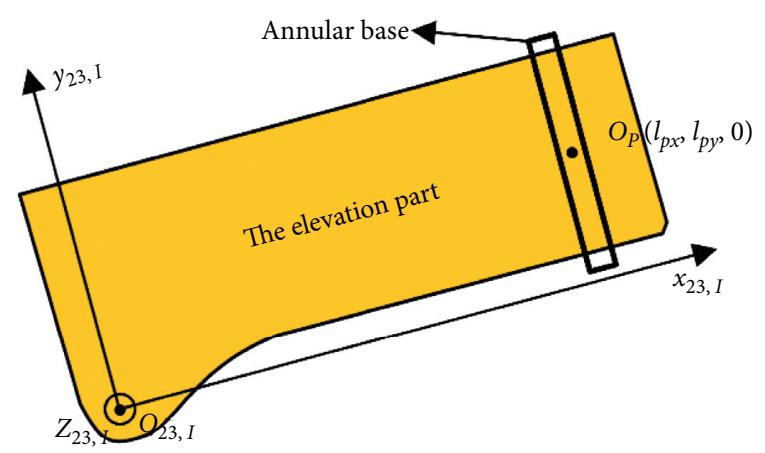

(b)

Figure 2: The design of the AAT: (a) the structure and (b) the location.

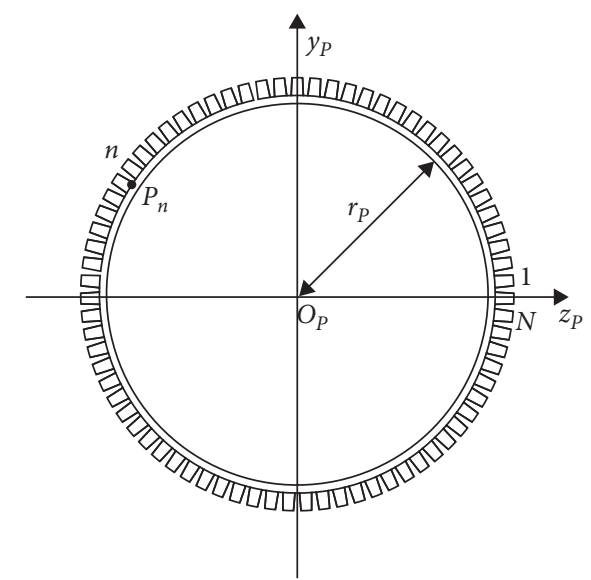

Figure 3: The schematic diagram of the AAT.

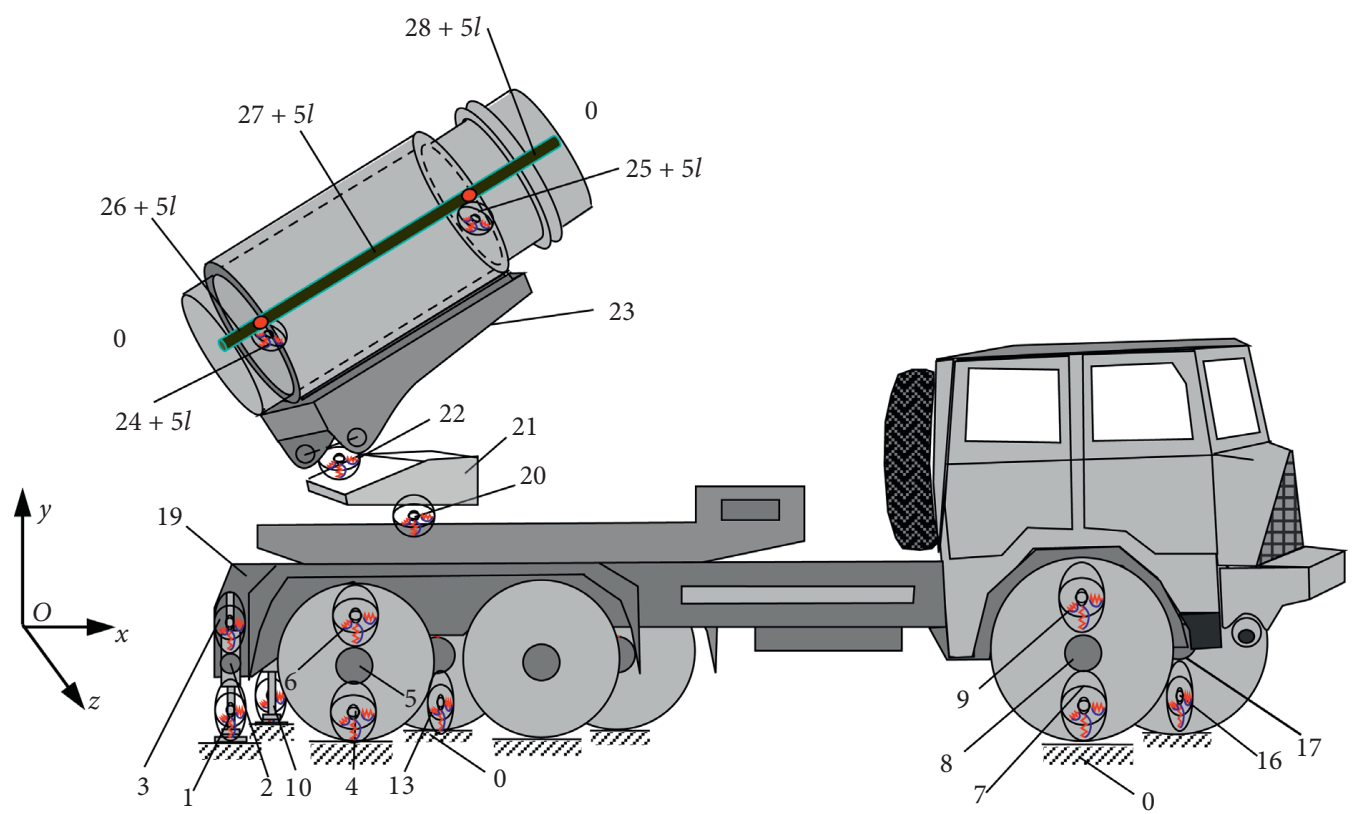

(a)

Figure 4: Continued. 


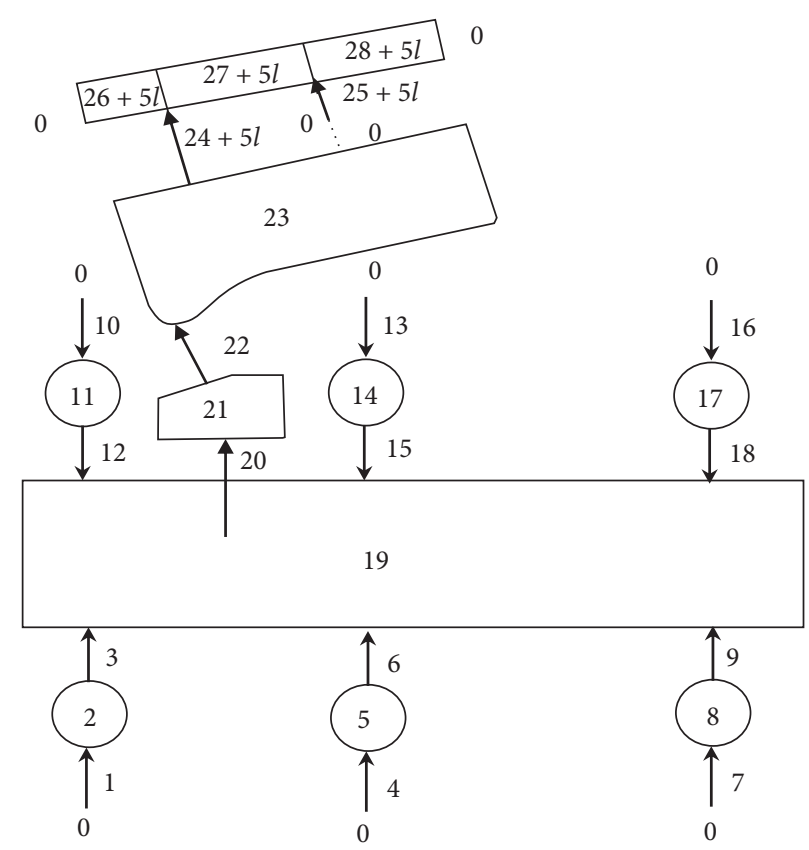

(b)

Figure 4: The model of the MLRS: (a) the dynamic model; (b) the topology.

which is very useful for the deduction of overall transfer equation in MSTMM. The topology of the MLRS dynamic model is presented in Figure 4(b), in which the dotted line denotes the "cutting point." There are 2 more boundary ends after "cutting" the close-loop subsystem, leading a corresponding geometric relationship [19].

Using the automatic deduction theorem, the overall transfer equation of MLRS can be automatically deduced according to Figure 4(b):

$$
U_{\text {all }} Z_{\text {all }}=0
$$

with

$$
Z_{\text {all }}=\left[Z_{28+5 l, 0}^{T}, Z_{1,0}^{T}, Z_{4,0}^{T}, Z_{7,0}^{T}, Z_{10,0}^{T}, Z_{13,0}^{T}, Z_{16,0}^{T}, Z_{26+5 l, 0}^{T}, Z_{23,25+5 l}^{T}\right]^{T} \text {, }
$$

being the state vectors of MLRS boundary ends, and

$$
U_{\text {all }}=\left[\begin{array}{ccccccccc}
-I_{12} & T_{1-28+5 l} & T_{4-28+5 l} & T_{7-28+5 l} & T_{10-28+5 l} & T_{13-28+5 l} & T_{16-28+5 l} & T_{26+5 l-28+5 l} & T_{23-28+5 l} C+T_{25+5 l-28+5 l} \\
O & G_{1-19} & G_{4-19} & O & O & O & O & O & O \\
O & G_{1-19} & O & G_{7-19} & O & O & O & O & O \\
O & G_{1-19} & O & O & G_{10-19} & O & O & O & O \\
O & G_{1-19} & O & O & O & G_{13-19} & O & O & O \\
O & G_{1-19} & O & O & O & O & G_{16-19} & O & O \\
O & G_{1-23} & G_{4-23} & G_{7-23} & G_{10-23} & G_{13-23} & G_{16-23} & O & G_{23-23} C \\
O & G_{1-27+5 l} & G_{4-27+5 l} & G_{7-27+5 l} & G_{10-27+5 l} & G_{13-27+5 l} & G_{16-27+5 l} & G_{26+5 l-27+5 l} & G_{23-27+5 l} C \\
O & G_{1-28+5 l} & G_{4-28+5 l} & G_{7-28+5 l} & G_{10-28+5 l} & G_{13-28+5 l} & G_{16-28+5 l} & G_{26+5 l-28+5 l} & G_{23-28+5 l} C+G_{25+5 l-28+5 l}
\end{array}\right],
$$

being the overall transfer matrix, where $T_{i-28+5 i}$ represents the successive premultiplication of all transfer matrices of elements in the transfer path from the boundary end $Z_{i, 0}$ to element $Z_{28+5 l, 0}$ and $G_{i-j}$ represents the successive premultiplication of all transfer matrices of elements in the transfer path from element $j$ to element $i$.

According to the boundary conditions, the reduced sate vector $\bar{Z}_{\text {all }}$ can be obtained by eliminating all zero elements in $Z_{\text {all }}$, and $U_{\text {all }}$ becomes a $60 \times 60$ square matrix $\bar{U}_{\text {all }}$ by removing the columns associated with the zero elements. Finally, (5) can be rewritten as

$$
\bar{U}_{\text {all }} \bar{Z}_{\text {all }}=0 .
$$

Then, the eigenfrequency equation of MLRS becomes

$$
\operatorname{det} \bar{U}_{\text {all }}=0 \text {. }
$$

The eigenfrequencies $\omega_{k}(k=1,2,3, \ldots, \infty)$ can be obtained solving the above equation. 
3.3. The Body Dynamics Equations. According to MSTMM, $M_{j}, C_{j}$, and $K_{j}$ are defined as the mass matrix, the damping matrix, and the stiffness matrix, respectively. $v_{j}$ is a column matrix composed of displacements (including angular displacements) which denotes the motion state of body element $j . v_{j, t}$ and $v_{j . t t}$ are the first and second derivatives of $v_{j}$ with respect to time $t$, respectively.

Considering the control action based on the thrusters' action, the body dynamic equation of the elevation part is as follows:

$$
M_{23} v_{23, t t}+C_{23} v_{23, t}+K_{23} v_{23}=f_{23}=\Phi_{\text {base }} u,
$$

where $\Phi_{\text {base }}=\left[\begin{array}{cccccc}0 & 1 & 0 & 0 & 0 & l_{p x} \\ 0 & 0 & 1 & l_{p y} & -l_{p x} & 0\end{array}\right]^{\mathrm{T}}$ is control position matrix decided by the AAT and $u=\left[\begin{array}{ll}u_{y} & u_{z}\end{array}\right]^{\mathrm{T}}$ is the control force with $u_{y}$ in $y$ direction and $u_{z}$ in $z$ direction of the bodyfixed frame of the elevation part $O_{23, I} x_{23, I} y_{23, I} z_{23, I}$.

The body dynamics equation of other body elements of MLRS is written as

$$
\begin{aligned}
& M_{j} v_{j, t t}+C_{j} v_{j, t}+K_{j} v_{j}=0, \\
& \quad(j=2,5,8,11,14,17,19,21,26+5 l, 27+5 l, 28+5 l) .
\end{aligned}
$$

Then, the body dynamics equation of the MLRS can be obtained by arranging the element body dynamic equations in order

$$
M v_{t t}+C v_{t}+K v=\Phi_{\text {base }} u
$$

where $M=\operatorname{diag}\left(M_{2}, \ldots, M_{27+5 l}, M_{28+5 l}\right), \quad C=\operatorname{diag}\left(C_{2}, \ldots\right.$, $\left.C_{27+5 l}, C_{28+5 l}\right)$, and $K=\operatorname{diag}\left(K_{2}, \ldots, K_{27+5 l}, K_{28+5 l}\right)$ are, respectively, the mass, damping, and stiffness augmented operators of the system. $v=\left[v_{2}^{T}, \ldots, v_{27+5 l}^{T}, v_{28+5 l}^{T}\right]^{\mathrm{T}}$ is the displacement column matrix.

3.4. The State Space Equation. The augmented eigenvector of MLRS consists of the displacement and angle mode shape of body elements corresponding to the $k^{\text {th }}$ eigenfrequency $\omega_{k}$ and is expressed as

$$
V^{k}=\left[V_{2}^{k T}, V_{5}^{k T}, V_{8}^{k T}, V_{11}^{k T}, V_{14}^{k T}, V_{17}^{k T}, V_{19}^{k T}, V_{21}^{k T}, V_{23}^{k T}, V_{26+5 l}^{k T}, V_{27+5 l}^{k T}, V_{28+5 l}^{k T}\right]^{T},
$$

where $V_{j}^{k}=\left[X_{j, O}^{k}, Y_{j, O}^{k}, Z_{j, O}^{k}, \Theta_{x j, O}^{k}, \Theta_{y j, O}^{k}, \Theta_{z j, O}^{k}\right]^{T}$.

According to the modal transformation, $v_{j}$ comes as

$$
v=\sum_{k=1}^{\infty} V^{k} q^{k}(t)
$$

where $q^{k}(t)$ is the $k^{\text {th }}$ generalized coordinates. Due to the existence of flexible bodies (element $27+5 l$ and $28+5 l$ ), the modal order is infinitely. Using the modal superposition method, an approximation of the MLRS dynamics responses can be expressed as

$$
\sum_{k=1}^{m} M V^{k} \ddot{q}^{k}(t)+\sum_{k=1}^{m} C V^{k} \dot{q}^{k}(t)+\sum_{k=1}^{m} K V^{k} q^{k}(t)=\Phi_{\text {base }} u \text {. }
$$

Supposing diagonal damping $C=\operatorname{diag}\left(C_{1}, C_{2}, \ldots\right)$ and taking the inner product in (15) with $V^{k}(k=1,2,3, \ldots)$ and the generalized coordinate equations of the MLRS can be obtained using the orthogonality [18]:

$$
\ddot{q}^{k}(t)+2 \zeta_{k} \omega_{k} \dot{q}^{k}(t)+\omega_{k}^{2} q^{k}(t)=b^{k} u,
$$

where $\zeta_{k}=C_{k} / 2 \omega_{k} M_{k}$ is the $k^{\text {th }}$ modal damping ratio, $M_{k}$ is the $k^{\text {th }}$ modal mass, and $b^{k}=\left\langle V^{k}, \Phi_{\text {base }}\right\rangle / M_{k}$.

The yawing and pitching angular velocity of the elevation part are defined as $\theta_{y}$ and $\theta_{z}$, respectively. They are chosen as the control output $y=\left[\begin{array}{ll}\dot{\theta}_{y} & \dot{\theta}_{z}\end{array}\right]^{\mathrm{T}}$ due to its influence on the rockets' initial influence [2].

Let

$$
x^{k}=\left[\begin{array}{c}
q^{k} \\
\dot{q}^{k}
\end{array}\right] \text {. }
$$

The state space equations of MLRS are described as

$$
\left\{\begin{array}{l}
\dot{x}=A x+B u \\
y=C x
\end{array}\right.
$$

where $x=\left[\begin{array}{c}x^{1} \\ x^{2} \\ \vdots \\ x^{n}\end{array}\right], \quad A=\operatorname{diag}\left(A^{1}, A^{2}, \ldots, A^{n}\right), \quad B=\left[\begin{array}{c}B^{1} \\ B^{2} \\ \vdots \\ B^{n}\end{array}\right]$, $C=\left[\begin{array}{llll}C^{1} & C^{2} & \ldots & C^{n}\end{array}\right], A^{k}=\left[\begin{array}{cc}0 & 1 \\ -\omega_{k}^{2} & -2 \zeta_{k} \omega_{k}\end{array}\right], B^{k}=\left[\begin{array}{c}0 \\ b^{k}\end{array}\right]$, $C^{k}=\left[\begin{array}{ll}0 & L^{k}\end{array}\right]$, and $L^{k}=\left[\begin{array}{ll}\Theta_{y 23, O}^{k} & \Theta_{z 23, O}^{k}\end{array}\right]^{\mathrm{T}}$.

\section{The Design of the Vibration Control}

4.1. The Hankel Model Reduction. For controller design, the model should be reduced, and the Hankel model reduction is chosen, which is from the perspective of quantitative descriptions of controllability and observability. The controllability and observability are quantified as controllability and observability Grammians:

$$
\begin{aligned}
& W_{c}(t)=\int_{0}^{t} e^{A \tau} B B^{T} e^{A^{T} \tau} \mathrm{d} \tau, \\
& W_{o}(t)=\int_{0}^{t} e^{A^{T} \tau} C^{T} C e^{A \tau} \mathrm{d} \tau .
\end{aligned}
$$

For a stable system, these two matrices can be obtained by solving the Lyapunov equations:

$$
\begin{aligned}
& A W_{c}+W_{c} A^{T}+B B^{T}=0, \\
& A^{T} W_{o}+W_{o} A+C^{T} C=0 .
\end{aligned}
$$

The Grammians depend on the choice of system state variable, while the eigenvalues of their product $W_{c} W_{o}$ are invariant. The $j^{\text {th }}$ eigenvalue is denoted as $\lambda_{i}\left(W_{c} W_{o}\right)$ and its square root is the Hankel singular value of the system. Then, 
a balanced state space realization $\left(A_{b}, B_{b}, C_{b}\right)$ for the system $(A, B, C)$ can be obtained through the linear transformation, such that the controllability and observability Grammians are equal and diagonal, and its diagonal entries are the Hankel singular values. The MATLAB function "balreal" can be used to compute the balanced state space realization and Hankel singular values of the system. Deleting the states with small Hankel singular values, the reduced model can be achieved:

$$
\left\{\begin{array}{l}
\dot{x}_{r}=A_{r} x_{r}+B_{r} u \\
y=C_{r} x_{r}
\end{array}\right.
$$

4.2. The LQR-PID Control Law. Due to its structure which is simple and easy to realize, PID control is commonly used in practice. Let $e=r-y$ and consider the PID control law as

$$
u=K_{p} e+K_{i} \int_{0}^{t} e d t+K_{d} \dot{e}
$$

where $r$ is the reference input vector and $K_{p}, K_{i}$, and $K_{d}$ are the gain matrices to be designed.

Let a new state variable be $\xi=\int_{0}^{t} e \mathrm{~d} t[24]$, then an augmented system is formed along with (21) given as

$$
\left\{\begin{array}{l}
\dot{z}=\bar{A} z+\bar{B} u+\bar{E} r \\
y=\bar{C} z
\end{array}\right.
$$

where $z=\left[\begin{array}{c}x_{r} \\ \xi\end{array}\right], \bar{A}=\left[\begin{array}{ll}A_{r} & 0 \\ C_{r} & 0\end{array}\right], \bar{B}=\left[\begin{array}{c}B_{r} \\ 0\end{array}\right], \bar{E}=\left[\begin{array}{l}0 \\ I\end{array}\right]$, and $\bar{C}=\left[\begin{array}{ll}C_{r} & 0\end{array}\right]$.

Substituting (21) into (22) and rearranging, one obtains

$$
\begin{aligned}
u & =\left(I-K_{d} C_{r} B_{r}\right)^{-1}\left[\left(K_{p} C_{r}+K_{d} C_{r} A_{r}\right)+K_{i} \xi\right] \\
& =\left[\begin{array}{lll}
\bar{K}_{p} & \bar{K}_{i} & \bar{K}_{d}
\end{array}\right] S z,
\end{aligned}
$$

where

$$
\begin{aligned}
\bar{K}_{p} & =\left(I-K_{d} C_{r} B_{r}\right)^{-1} K_{p}, \\
\bar{K}_{i} & =\left(I-K_{d} C_{r} B_{r}\right)^{-1} K_{i}, \\
\bar{K}_{d} & =\left(I-K_{d} C_{r} B_{r}\right)^{-1} K_{d}, \\
S & =\left[\begin{array}{cc}
C_{r} & 0 \\
C_{r} A_{r} & 0 \\
0 & I
\end{array}\right] .
\end{aligned}
$$

Let $K=\left[\begin{array}{lll}\bar{K}_{p} & \bar{K}_{i} & \bar{K}_{d}\end{array}\right] S$; the state feedback control for system (23) is $u=K z$ which can be obtained by minimizing the quadratic performance cost:

$$
J=\int_{0}^{\infty}\left(z^{T} Q z+u^{T} R u\right) \mathrm{d} t
$$

where $Q=Q^{T} \geq 0$ is the state weighted matrix and $R=R^{T}>0$ is the control weighted matrix. And it is obtained by solving the algebraic Riccati equation with $r=0$ :

$$
\bar{A}^{T} P+P \bar{A}-P \bar{B} R^{-1} \bar{B}^{T} P+Q=0,
$$

for matrix $P=P^{T}>0$. Then, the optimal gain vector becomes

$$
K=-R^{-1} \bar{B}^{T} P
$$

which can be easily solved using the MATLAB command "lqr."

Then, the PID parameters turn to

$$
\begin{aligned}
& K_{d}=\bar{K}_{d}\left(I+C_{r} B_{r} \bar{K}_{d}\right)^{-1}, \\
& K_{p}=\left(I-K_{d} C_{r} B_{r}\right) \bar{K}_{p}, \\
& K_{i}=\left(I-K_{d} C_{r} B_{r}\right) \bar{K}_{i} .
\end{aligned}
$$

4.3. The Management of the AAT. The PID control law assumes that the actuators can continuously produce the desired control force. However, the thrusters can only be used once to provide an approximately constant impulsive force with a constant duration. As shown in Figure 5, if the impulse of control force $U$ during some period $\left[t_{0}, t_{1}\right]$ is equal to that of a thruster, the control force $U$ can be replaced with the force of the thruster since they produce the same impulse [25].

With the AAT, a novel management of the AAT for discontinuous impulsive force approximation of the continuous control force is introduced. According to the special arrangement of the AAT, two parameters must be determined to fire a thruster in the AAT. The firing time of a thruster is symbolized as $t$ and the corresponding number of the thruster to be fired in the AAT is symbolized as $n$. These parameters are illustrated as follows.

Consider the impulse of the control force and the thruster will be fired when the resultant impulse of $u_{y}$ and $u_{z}$ is greater than the impulse of the thruster as shown in Figure 5. So a thruster will be fired when

$$
\sqrt{\left(\int_{t_{0}}^{t} u_{y} d t\right)^{2}+\left(\int_{t_{0}}^{t} u_{z} d t\right)^{2}} \geq F_{P} \Delta t_{P}
$$

where $u_{y}$ and $u_{z}$ are the control force generated by the PID law, $F_{P}$ and $\Delta t_{P}$ are the magnitude and duration of the thruster impulse force, and $t_{0}$ and $t$ are the start and end times for impulse calculation. It should be noted that the value of $t_{0}$ is reset when a thruster is fired and $t$ is the time in which inequality (31) is satisfied.

Based on the annularly arrangement of the AAT shown in Figure 3 and the force expression in (3), the firing number $n$ is determined by the impulse of $u_{y}$ and $u_{z}$ when the inequality in (31) is satisfied: 


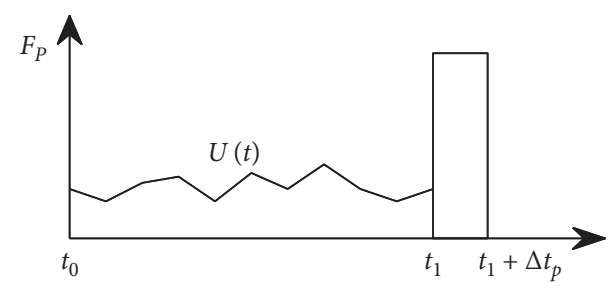

FIgURE 5: Thruster equivalent impulse approximation of the control force impulse.

$$
\left\{n_{0}=\operatorname{round}\left(\frac{\arctan \left(\left(\int_{t_{0}}^{t} u_{y} \mathrm{~d} t\right) /\left(\int_{t_{0}}^{t} u_{z} \mathrm{~d} t\right)\right)+\pi}{2 \pi / N}\right)\right\} \text {. }
$$

As the thrusters are single use, whether the thruster is available or not should be determined before it is fired. If the mark of thruster availability $S_{n_{0}}$ is 1 , the firing number $n=n_{0}$. If not, the firing number $n$ should be searched as follows, which helps restrain the angular vibration:

$$
\begin{aligned}
& \min \quad\left(a b s\left(n-n_{0}\right)\right) \\
& \text { s.t. } \quad S_{n}==1 \\
& n \in\left[n_{0}-\frac{N}{8}, n_{0}+\frac{N}{8}\right] .
\end{aligned}
$$

The controller will not work if there is no thruster available in the search range.

\section{Simulation}

5.1. The Simulation System and Parameters. The jet force and contact force between the rocket and launcher are the source of disturbance for the MLRS. And the dynamic response of the MLRS under these disturbances is specifically described in [1] and is omitted in this paper. A certain type of MLRS with 18 tubes is taken as the example in this paper, and the simulation system for MLRS vibration control in the launching with the AAT is established as shown in Figure 6. The thrusters number of the AAT is $N=72$ in this paper.

The reduced model $\left(A_{r}, B_{r}, C_{r}\right)$ turns to four states by deleting the states with the Hankel singular values smaller than $2 \times 10^{-5}$. The parameters of LQR are chosen as $Q=$ $I_{6} \times 10^{3}$ and $R=I_{2} \times 10^{-3}$. Then, the value of PID turns to be

$$
\begin{aligned}
K_{p} & =\left[\begin{array}{cc}
4.07 \times 10^{3} & -2.89 \times 10^{5} \\
3.00 \times 10^{5} & -8.26 \times 10^{3}
\end{array}\right], \\
K_{i} & =\left[\begin{array}{cc}
-3.06 \times 10^{3} & 5.64 \times 10^{3} \\
-8.95 \times 10^{3} & -4.52 \times 10^{3}
\end{array}\right], \\
K_{d} & =\left[\begin{array}{ll}
-18.12 & 713.92 \\
-723.96 & -12.70
\end{array}\right] .
\end{aligned}
$$

5.2. Results and Discussion. Considering the quantitative limitation of thrusters, the control action is limited in the period which begins when the rocket's front band leaves the muzzle and finishes when the rear band leaves the muzzle. Setting the ignition time of a rocket to be 0 , the time when the rocket's front band leaves the muzzle is $60 \mathrm{~ms}$ and the time when the rear band leaves the muzzle is $118 \mathrm{~ms}$ accordingly. The amplitude of the elevation part's resultant angular velocity of $y$ and $z$ direction is denoted as $\dot{\theta}_{y z}$. The RMS value of $\theta_{y z}$ in the control period is considered as the performance evaluation index of the control system. The conditions of the simulation are listed as follows.

Condition 1. Parameters of the thruster in the AAT are $F_{P}=2000 \mathrm{~N}, \Delta t_{P}=10 \mathrm{~ms}, \Delta t_{r}=3 \mathrm{~ms}$, and $\Delta t_{d}=3 \mathrm{~ms}$. Firing time interval of any two successive rockets is 1 second.

Condition 2. Parameters of the thruster in the AAT are $F_{P}=5000 \mathrm{~N}, \Delta t_{P}=10 \mathrm{~ms}, \Delta t_{r}=3 \mathrm{~ms}$, and $\Delta t_{d}=3 \mathrm{~ms}$. Firing time interval of any two successive rockets is 1 second.

5.2.1. Analysis of Condition 1. The RMS value of $\dot{\theta}_{y z}$ in the control period for each rocket under Condition 1 is compared with those without control in Table 1. The results show that the vibration of the elevation part's angular velocity is reduced by the $2000 \mathrm{~N}$-thrusters. The average reduction percentage is $19.32 \%$. During the 1st rocket's control period, the elevation part's vibration is the smallest and reduced by $41.47 \%$, because the MLRS is still before the 1st rocket is ignited. It can be seen from Table 1 that there is nearly no effect during the 8 th and 13th rocket' control period, because the available thrusters in the AAT for the control demand are used in the former control period. The simulated angular velocities of the elevation part in 2 nd and 6th rocket's launching process are showed in Figures 7 and 8, respectively.

5.2.2. Analysis of Condition 2. The RMS value of $\dot{\theta}_{y z}$ in the control period for each rocket under Condition 2 is compared with those without control in Table 2. Compared with the results under Condition 1, the better control effectiveness is accomplished under Condition 2. The average reduction percentage is $31.43 \%$, and the best one is as highly as $46.43 \%$. For the 1st rocket, the threshold in (31) is not reached and the AAT does not work due to the higher magnitude of the thrusters under Condition 2 and the elevation part's vibration is the small during the control period. It can be seen from Table 2 that there is a little effect during the 13th rocket' control period because of the same reason of no available thrusters. The simulated angular velocities of the elevation part in 6th and 14th rocket's launching process are showed in Figures 9 and 10, respectively. 


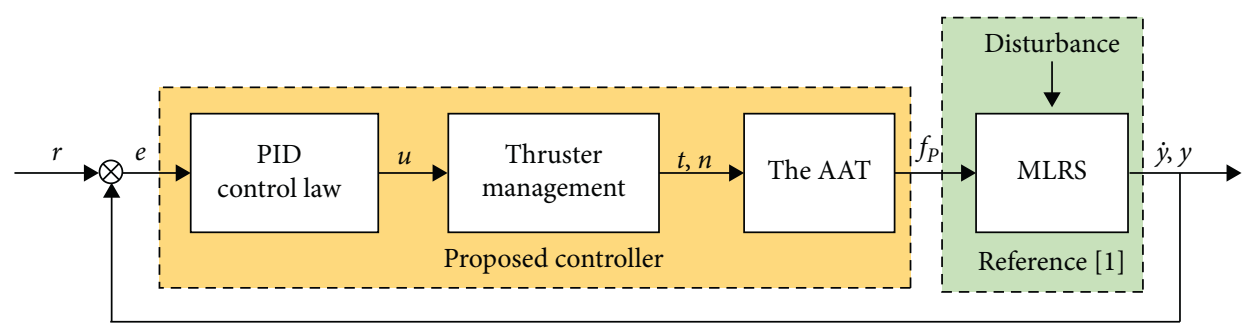

FIgURE 6: Simulation system for MLRS vibration control in the launching with the AAT.

TABLe 1: Comparison of $\dot{\theta}_{y z}$ in the control period under Condition 1.

\begin{tabular}{|c|c|c|c|}
\hline The rocket order & Without control & With control under Condition 1 & Reduction percentage (\%) \\
\hline 1 & 0.0038 & 0.0022 & 41.47 \\
\hline 2 & 0.0143 & 0.0105 & 26.20 \\
\hline 3 & 0.0136 & 0.0108 & 20.24 \\
\hline 4 & 0.0161 & 0.0139 & 13.62 \\
\hline 5 & 0.0143 & 0.0105 & 26.50 \\
\hline 6 & 0.0206 & 0.0160 & 22.27 \\
\hline 7 & 0.0192 & 0.0151 & 21.64 \\
\hline 8 & 0.0165 & 0.0166 & -0.58 \\
\hline 9 & 0.0257 & 0.0209 & 18.71 \\
\hline 10 & 0.0181 & 0.0163 & 9.86 \\
\hline 11 & 0.0243 & 0.0197 & 19.22 \\
\hline 12 & 0.0165 & 0.0148 & 10.27 \\
\hline 13 & 0.0194 & 0.0192 & 0.82 \\
\hline 14 & 0.0155 & 0.0122 & 20.78 \\
\hline 15 & 0.0182 & 0.0145 & 20.12 \\
\hline 16 & 0.0269 & 0.0185 & 31.45 \\
\hline 17 & 0.0220 & 0.0159 & 27.87 \\
\hline 18 & 0.0126 & 0.0085 & 32.40 \\
\hline The average value & 0.1765 & 0.01424 & 19.32 \\
\hline
\end{tabular}

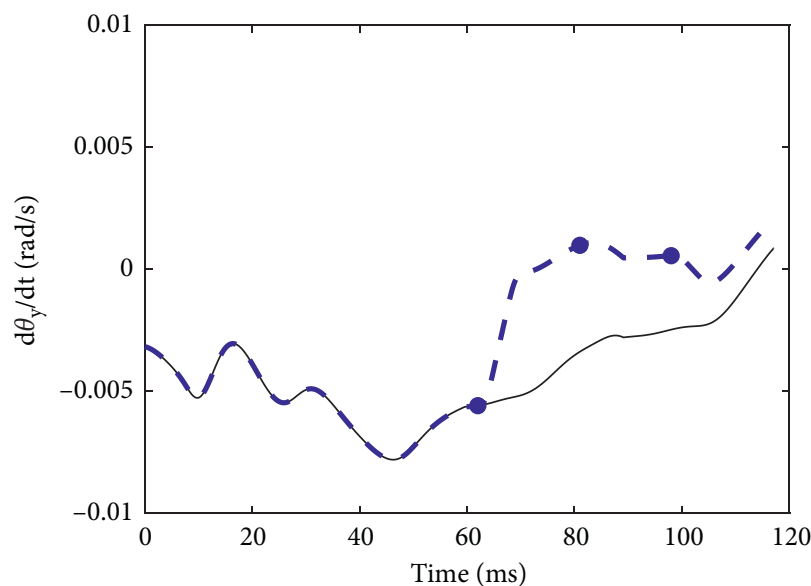

- Without control

-..- With control

(a)

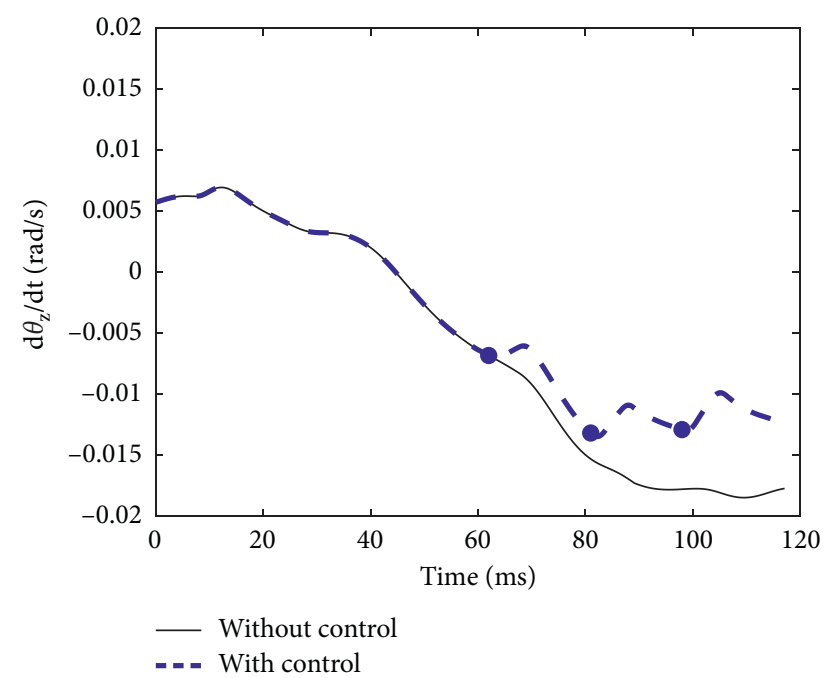

(b)

FIGURE 7: Simulated results of the elevation part in launching process for the 2nd rocket under Condition 1: (a) the yawing angular velocity and (b) the pitching angular velocity. The blue dots indicate the action of the AAT. 


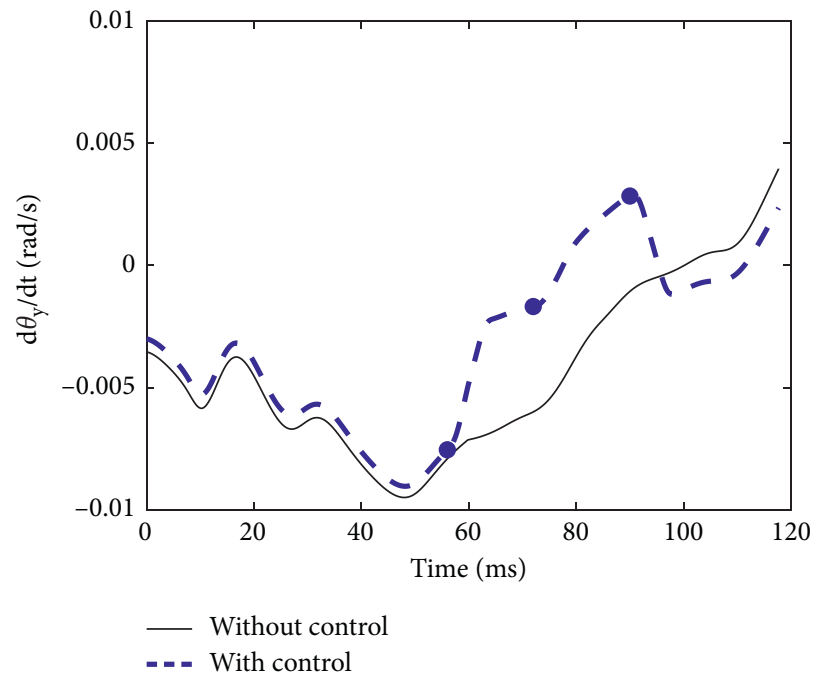

(a)

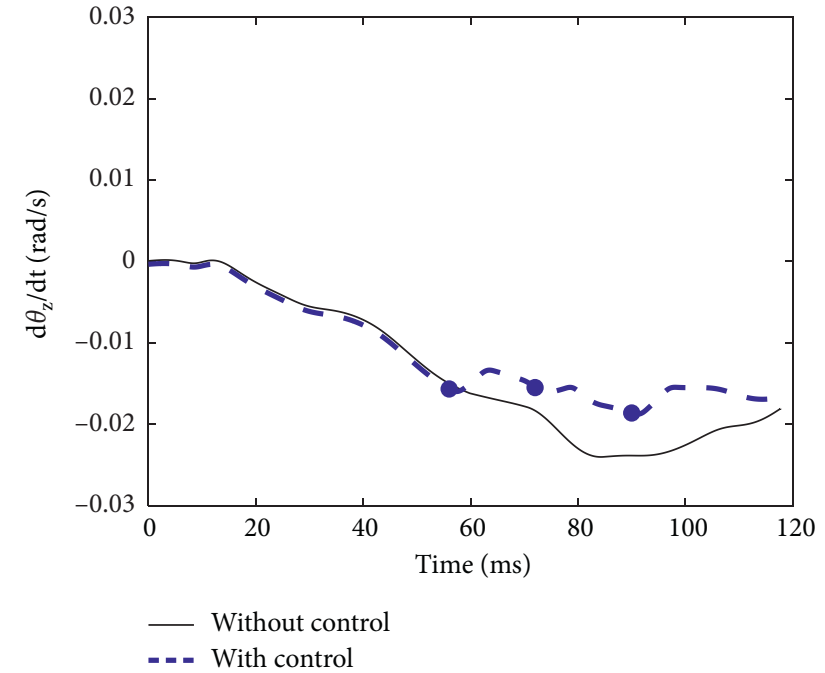

(b)

FIGURE 8: Simulated results of the elevation part in launching process for the 6th rocket under Condition 1: (a) the yawing angular velocity and (b) the pitching angular velocity. The blue dots indicate the action of the AAT.

TABle 2: Comparison of $\dot{\theta}_{y z}$ in the control period under Condition 2.

\begin{tabular}{lccc}
\hline The rocket order & Without control & With control under Condition 1 & Reduction percentage (\%) \\
\hline 1 & 0.0038 & 0.0038 & 0 \\
2 & 0.0143 & 0.0099 & 30.35 \\
3 & 0.0136 & 0.0094 & 30.66 \\
4 & 0.0161 & 0.0101 & 37.29 \\
5 & 0.0143 & 0.0098 & 31.55 \\
6 & 0.0206 & 0.0138 & 33.14 \\
7 & 0.0192 & 0.0121 & 37.17 \\
8 & 0.0165 & 0.0088 & 46.43 \\
9 & 0.0257 & 0.0117 & 54.96 \\
10 & 0.0181 & 0.0140 & 22.79 \\
11 & 0.0243 & 0.0161 & 33.74 \\
12 & 0.0165 & 0.0117 & 29.45 \\
13 & 0.0194 & 0.0181 & 6.70 \\
14 & 0.0155 & 0.0091 & 40.87 \\
15 & 0.0182 & 0.0148 & 18.56 \\
16 & 0.0269 & 0.0167 & 37.77 \\
17 & 0.0220 & 0.0132 & 40.28 \\
18 & 0.0126 & 0.0096 & 23.23 \\
The average value & 0.01765 & 0.0121 & 31.43 \\
\hline
\end{tabular}

\section{Experiment}

6.1. The Experiment Setup. According to the analysis in Section 5, the $5000 \mathrm{~N}$-thrusters are chosen for the AAT due to the better simulation results. The prototype of the proposed control system is shown in Figure 11. The proposed system is consisted of the Data Signal Processing (DSP), the Inertial Measurement Unit (IMU), the AAT, and the MLRS. The angular velocities of the elevation part are the control output of the proposed system, so the IMU (SDI-GC7050C, $1000 \mathrm{~Hz}$ ) is chosen as the sensor in the system and it is mounted on the platform of the elevation part. The DSP (SOM-TL6748, 456 MHz) is the key of the proposed system. It completes the functions of data acquisition with the sampling rate of $1000 \mathrm{~Hz}$, the ignition of the rockets, and the control of the AAT. The angular signal (including angular motion and angular velocity) of the elevation part measured by the IMU is sent to the DSP through RS422 serial port and recorded in the DSP. The rockets are ignited in sequence by the DSP with the ignition signal recorded in the DSP. During the control period after the rocket ignited, the thrusters in the AAT will be fired by the DSP through relays when the vibration control in the DSP is satisfied based on the angular signal of the IMU. The ignition of the AAT is also recorded in the DSP. 


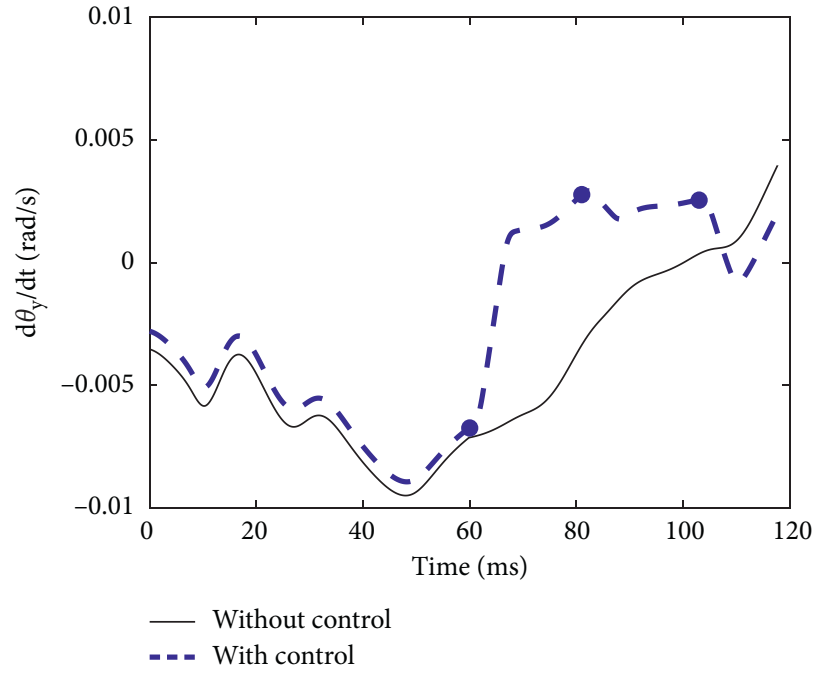

(a)

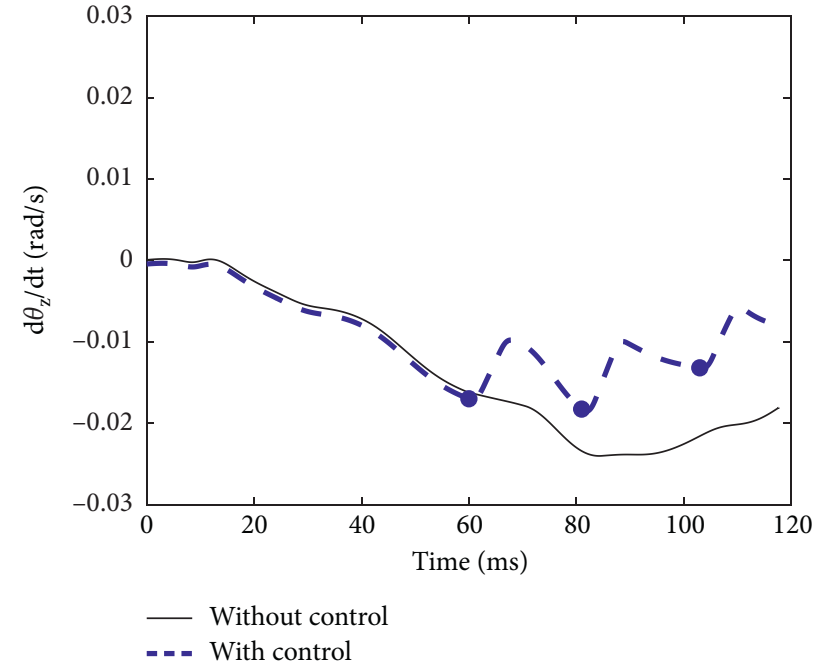

(b)

FIGURE 9: Simulated results of the elevation part in launching process for the 6th rocket under Condition 2: (a) the yawing angular velocity and (b) the pitching angular velocity. The blue dots indicate the action of the AAT.

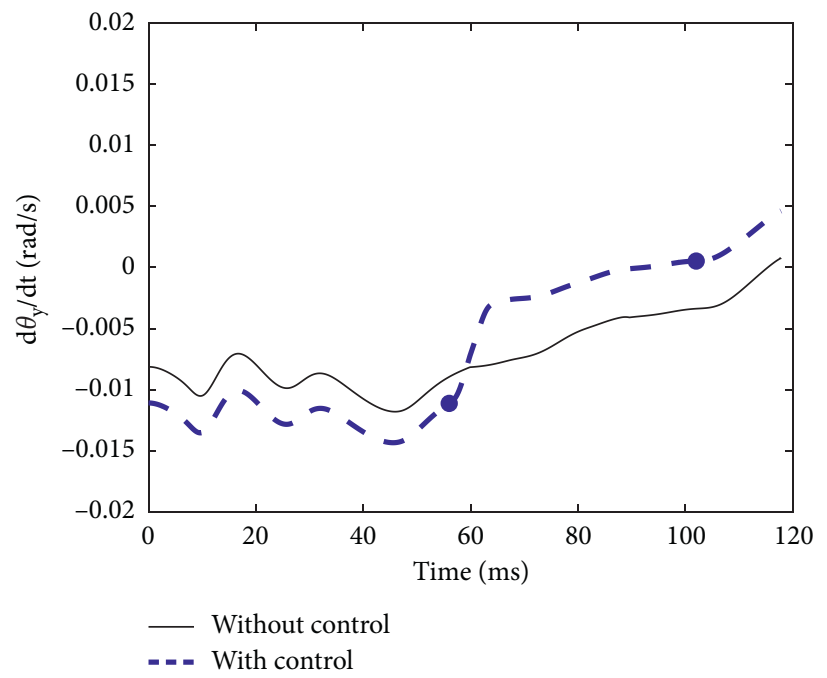

(a)

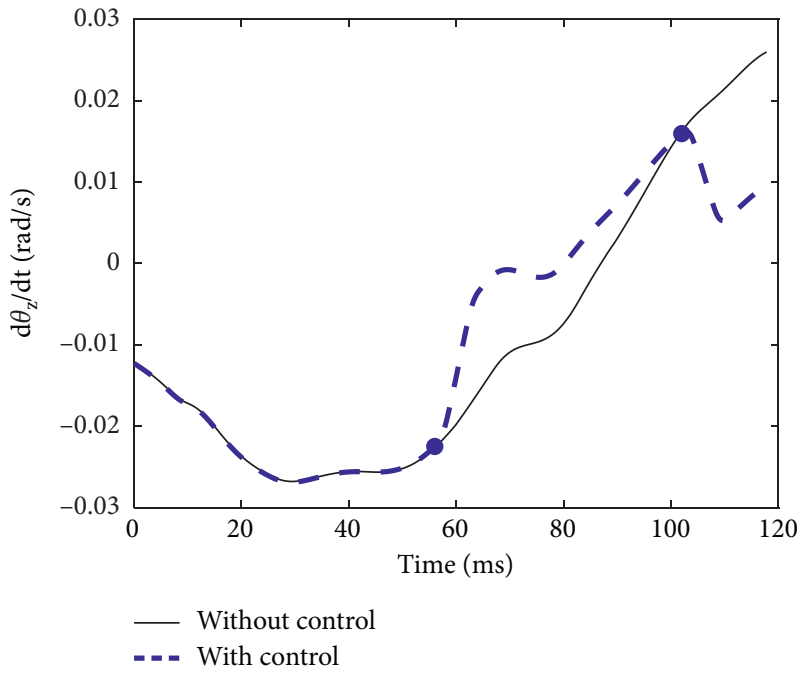

(b)

FIGURE 10: Simulated results of the elevation part in launching process for the 14th rocket under Condition 2: (a) the yawing angular velocity and (b) the pitching angular velocity. The blue dots indicate the action of the AAT.

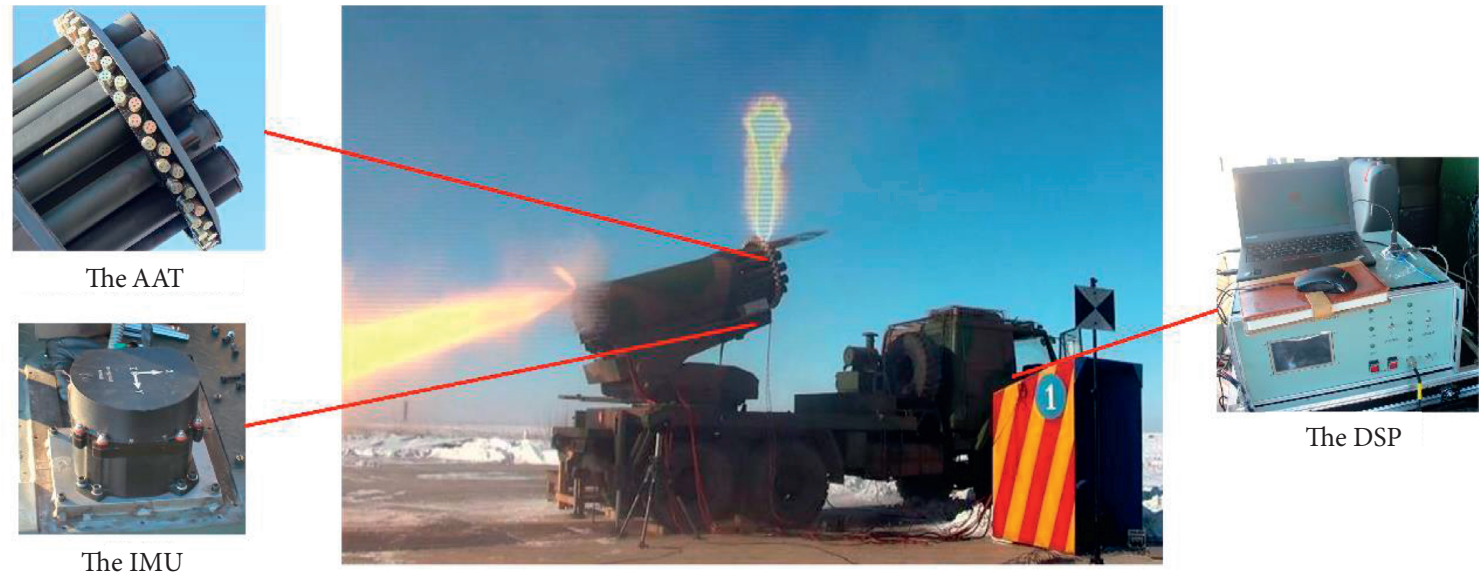

The MLRS

FIGURE 11: The prototype of the proposed control system. 
TABLE 3: Comparison of the maximum value of $\dot{\theta}_{y z}$ in the control period.

\begin{tabular}{lccc}
\hline The rocket order & Without control & With control & Reduction percentage (\%) \\
\hline 1 & 0.0204 & 0.0207 & - \\
2 & 0.0451 & 0.0336 & 25.47 \\
3 & 0.0418 & 0.0242 & 42.14 \\
\hline
\end{tabular}

TABLE 4: Comparison of the RMS value of $\dot{\theta}_{y z}$ in the control period.

\begin{tabular}{lccc}
\hline The rocket order & Without control & With control & Reduction percentage (\%) \\
\hline 1 & 0.0121 & 0.0114 & - \\
2 & 0.0209 & 0.0127 & 39.37 \\
3 & 0.0204 & 0.0123 & 39.90 \\
\hline
\end{tabular}

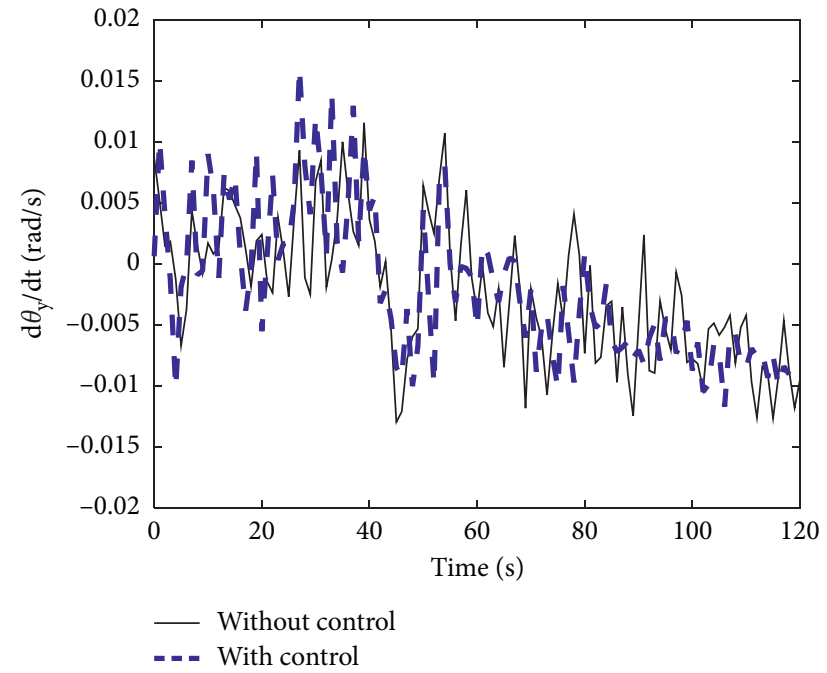

(a)

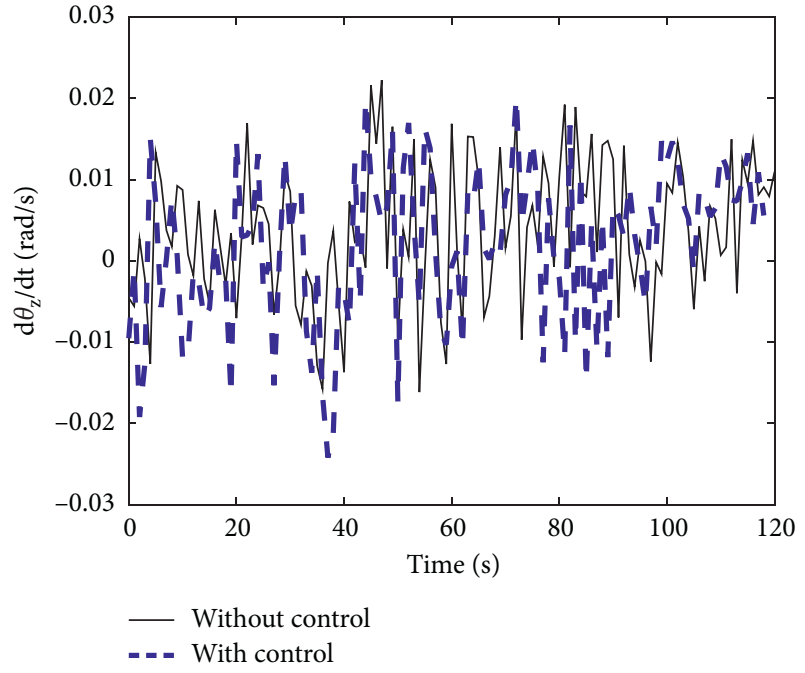

(b)

FIGURE 12: Experimental results of the elevation part in launching process for the 1st rocket: (a) the yawing angular velocity and (b) the pitching angular velocity.

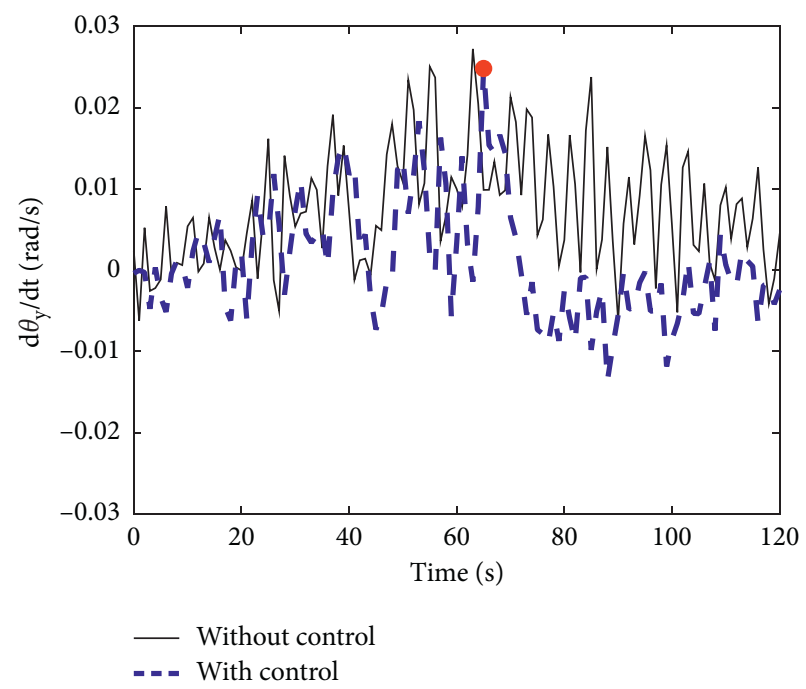

(a)

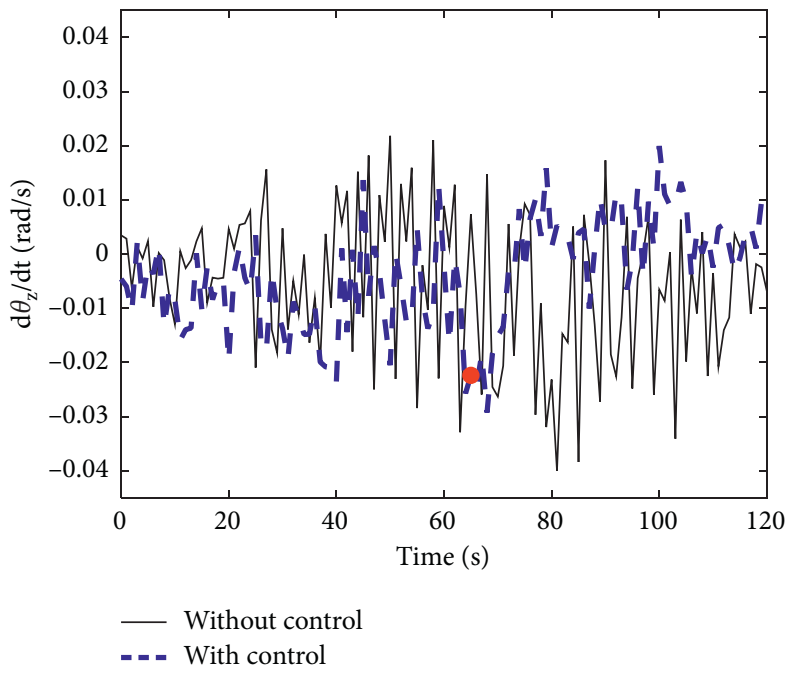

(b)

FIGURE 13: Experimental results of the elevation part in launching process for the 2 nd rocket: (a) the yawing angular velocity and (b) the pitching angular velocity. The red dots indicate the action of the AAT. 


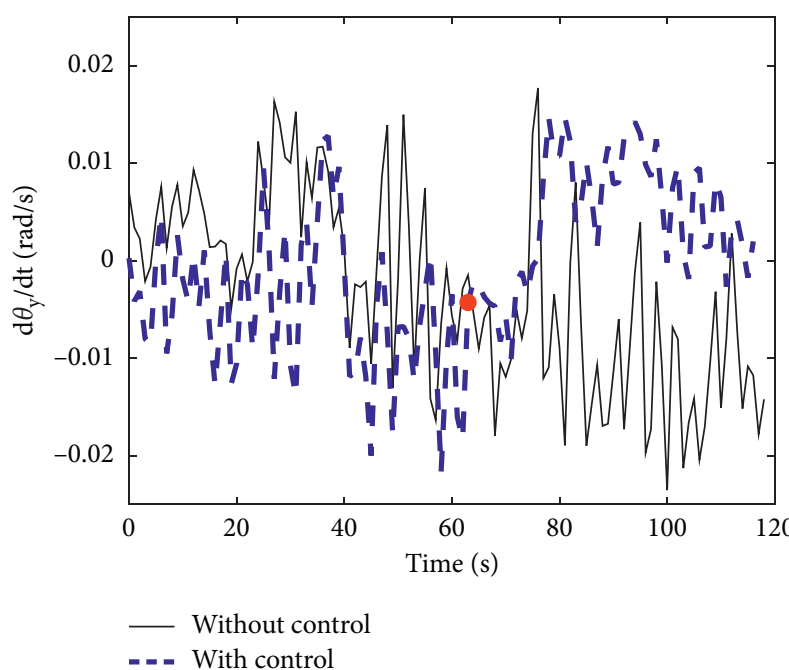

(a)

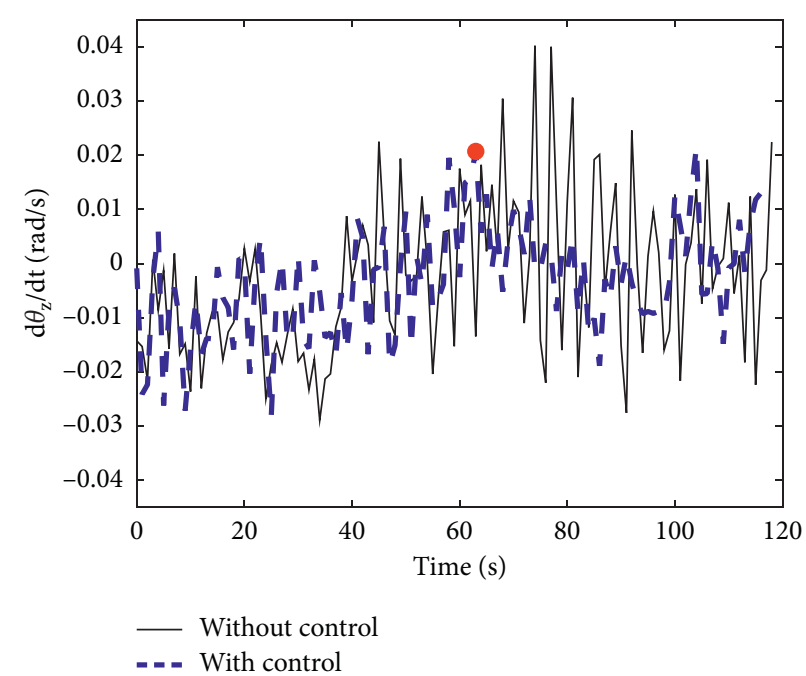

(b)

FIGURE 14: Experimental results of the elevation part in launching process for the 3rd rocket: (a) the yawing angular velocity and (b) the pitching angular velocity. The red dots indicate the action of the AAT.

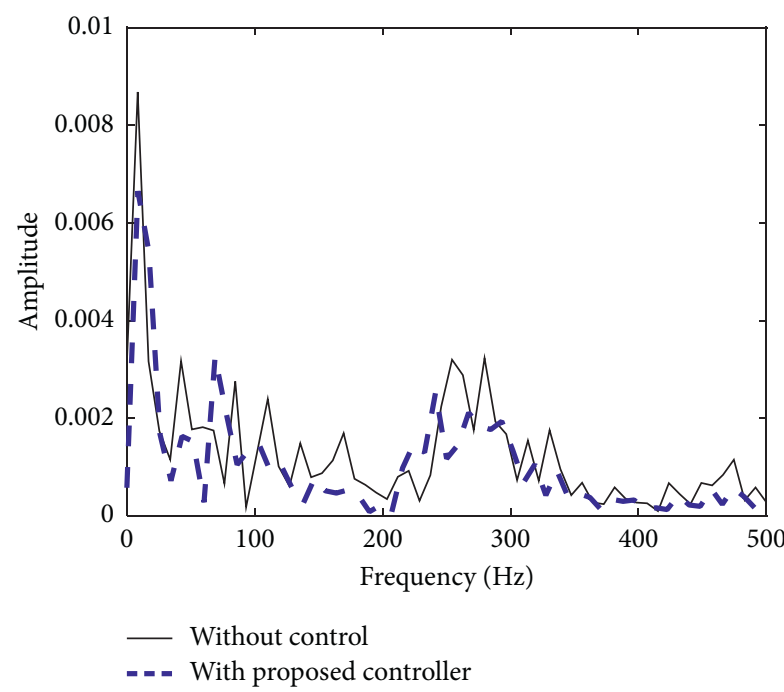

(a)

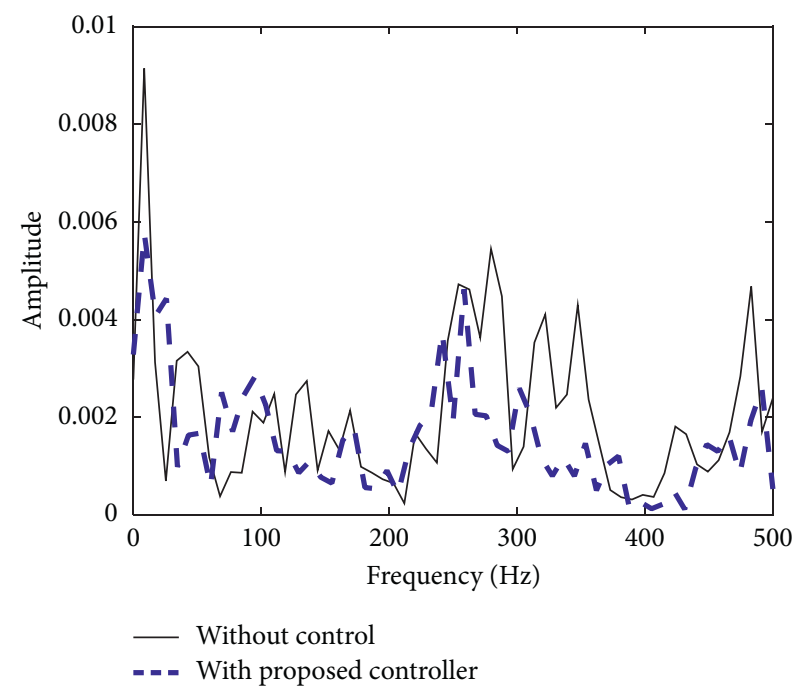

(b)

FIGURE 15: Experimental results of the elevation part in launching process for the 3rd rocket in frequency domain: (a) the yawing angular velocity and (b) the pitching angular velocity.

6.2. Results and Discussion. Two groups of experiments of a salvo of three rockets, in which one is applied without control and the other one with control, are carried out. Tables 3 and 4, respectively, compare the maximum value and the RMS value of $\dot{\theta}_{y z}$ in the control period. The RMS values $\dot{\theta}_{y z}$ for the 2 nd and 3 rd rocket are, respectively, $39.37 \%$ and $39.9 \%$ lower than those without control. It turns out that the vibration of the MLRS is effectively reduced by the proposed control system. The study provides a new idea to improve the dispersion characteristic by attenuating the vibration of MLRS in launching process.

Figures 12-14, respectively, show the experiment results in launching process for each rocket, in which the time axis setting is the same as the simulation results. From Figure 12, there is no pulse thruster fired due to the fact that the angular velocities without control for the first rocket are smaller, which is consistent with the simulation results. 46th thruster is fired on $62 \mathrm{~ms}$ in Figures 10 and 13 and thruster is fired on $64 \mathrm{~ms}$ in Figure 14. In order to better visualize the vibration suppression of the proposed system, the experiment results in launching process for the 3rd rocket are analyzed using FFT and shown in Figure 15.

\section{Conclusion}

A novel vibration control system applying the AAT for MLRS in launching process was presented and the control effectiveness has been studied by simulation and experiment in this paper. The main conclusions can be drawn as follows: 
(1) A novel vibration control system applying the AAT for MLRS in launching process was designed and built. A new arrangement of thrusters was designed and applied to the vibration control of MLRS in the short launching process.

(2) The control law and the management for the AAT proposed in this paper are effective to realize the vibration control of the MLRS in the launching process. Under Condition 2, the elevation part's vibration is reduced by $31.43 \%$ on average by simulation.

(3) The proposed system is verified effectively by experiments of a salvo of 3 rockets and the best vibration reduction percentage is up to 39.90 .

\section{Data Availability}

Due to legal and ethical concerns, especially third-party rights and commercial confidentiality, the data are not provided.

\section{Conflicts of Interest}

The authors declare that there are no conflicts of interest regarding the publication of this paper.

\section{Acknowledgments}

This work was supported by National Natural Science Foundation of China (no. 11972193).

\section{References}

[1] X. T. Rui, Y. Q. Lu, G. P. Wang, W. D. Chen, and L. F. Yun, Simulation and Test Methods of Launch Dynamics of Multiple Launch Rocket System, National Defense Industry Press, Beijing, China, 2003.

[2] W. B. Tang, X. T. Rui, G. P. Wang, and F. F. Yang, "Quantitative analysis for affecting factors of firing dispersion of multiple launch rockets system," Journal of Nanjing University of Science and Technology, vol. 39, pp. 704-710, 2015.

[3] J. E. Cochran, R. T. Gunnels, and R. T. J. McCutchen, Launchers as Passive Controllers, Interim Report, Auburn University, Auburn, AL, USA, 1980.

[4] J. J. Cochran and D. Christensen, "Launcher/rocket dynamics and passive control," in Proceedings of the 7th Atmospheric Flight Mechanics Conference, Albuquerque, NM, USA, August 1981.

[5] J. Cochran and R. Gunnels, "Rocket launchers as passive controllers," Final Report, Auburn University, Auburn, AL, USA, 1981.

[6] G. G. Chen and W. X. Zhao, "A study on mechanism of rocket passive controller," Journal of Ballistics, vol. 3, pp. 61-64, 1991.

[7] W. X. Zhao and G. G. Chen, "Rocket passive controller," Acta Armamentarii, vol. 17, pp. 40-45, 1996.

[8] Y. Zhang, Y. Jing, and S. Zhang, "Robust decentralized reliable control for uncertain interconnected delayed systems," IFAC Proceedings Volumes, vol. 35, no. 1, pp. 139-143, 2002.

[9] Z. Q. Xu, Y. Feng, and C. G. Yu, "Variable damping dynamic response control on impact vibration of rocket launcher using hydraulic system," Machine Tool and Hydraulics, vol. 37, pp. 106-109, 2009.
[10] K. Dokumaci, M. T. Aydemir, and M. U. Salamci, IEEE, Modeling, PID Control and Simulation of a Rocket Launcher System, IEEE, New York, NY, USA, 2014.

[11] B. Li and X. Rui, "Vibration control of uncertain multiple launch rocket system using radial basis function neural network," Mechanical Systems and Signal Processing, vol. 98, pp. 702-721, 2018.

[12] Y. Li and N. Qi, "Logic-based guidance law for interceptor missiles steered by aerodynamic lift and divert thruster," IEEE Transactions on Control Systems Technology, vol. 19, no. 4, pp. 884-890, 2011.

[13] X. Yang and H. Gao, "Robust reliable control for autonomous spacecraft rendezvous with limited-thrust," Aerospace Science and Technology, vol. 24, no. 1, pp. 161-168, 2013.

[14] T. W. Lim, "Thruster attitude control system design and performance for tactical satellite 4 maneuvers," Journal of Guidance, Control, and Dynamics, vol. 37, no. 2, pp. 403-412, 2014.

[15] Z. Zhan, X. Rui, G. Wang, F. Yang, and J. He, "Vibration control for launcher of multiple launch rocket system based on transfer matrix method of multibody system," Acta Mechanica Sinica, vol. 42, pp. 583-590, 2010.

[16] L. L. Gu, X. T. Rui, G. P. Wang, B. Li, and T. X. Tu, “Asme, vibration controller optimization design for multiple launch rocket system with pulse thrusters based on transfer matrix method for multibody systems," in Proceedings of the 14th International Conference on Multibody Systems, Nonlinear Dynamics, and Control, Quebec, Canada, 2018.

[17] X. Rui, X. Wang, Q. Zhou, and J. Zhang, "Transfer matrix method for multibody systems (rui method) and its applications," Science China Technological Sciences, vol. 62, no. 5, pp. 712-720, 2019.

[18] X. T. Rui, G. P. Wang, and J. S. Zhang, Transfer Matrix Method for Multibody Systems: Theory and Applications, Wiley, Hoboken, NJ, USA, 2018.

[19] X. T. Rui, J. S. Zhang, and Q. B. Zhou, "Automatic deduction theorem of overall transfer equation of multibody system," Advances in Mechanical Engineering, vol. 6, 2014.

[20] X. Rui, G. Wang, Y. Lu, and L. Yun, "Transfer matrix method for linear multibody system," Multibody System Dynamics, vol. 19, no. 3, pp. 179-207, 2008.

[21] G. P. Wang, X. T. Rui, and W. B. Tang, "Active vibration control design method based on transfer matrix method for multibody systems," Journal of Engineering Mechanics, vol. 143, no. 6, 2017.

[22] G. Chen, X. Rui, L. K. Abbas, G. Wang, F. Yang, and W. Zhu, "A novel method for the dynamic modeling of stewart parallel mechanism," Mechanism and Machine Theory, vol. 126, pp. 397-412, 2018.

[23] B. Rong, X. Rui, K. Lu, L. Tao, G. Wang, and F. Yang, "Dynamics analysis and wave compensation control design of ship's seaborne supply by discrete time transfer matrix method of multibody system," Mechanical Systems and Signal Processing, vol. 128, pp. 50-68, 2019.

[24] J. K. Pradhan and A. Ghosh, "Multi-input and multi-output proportional-integral-derivative controller design via linear quadratic regulator-linear matrix inequality approach," IET Control Theory \& Applications, vol. 9, no. 14, pp. 2140-2145, 2015.

[25] H. Ashrafiuon, "Guidance and attitude control of unstable rigid bodies with single-use thrusters," IEEE Transactions on Control Systems Technology, vol. 25, no. 2, pp. 401-413, 2017. 